\title{
Moderate/severe Depression (MADRS) Can Affect the Quality of Life and Outcome Among Patients Admitted to Breast Cancer Diagnosis Unit
}

\author{
MATTI ESKELINEN ${ }^{1}$, TUOMAS SELANDER ${ }^{2}$, PAULA OLLONEN ${ }^{3}$ and RIIKA KORHONEN ${ }^{1}$ \\ Departments of ${ }^{1}$ Surgery and ${ }^{3}$ Psychiatry and ${ }^{2}$ Science Service Center, Kuopio University Hospital, \\ and School of Medicine, University of Eastern Finland, Kuopio, Finland
}

\begin{abstract}
Aim: The Montgomery-Asberg Depression Rating Scale (MADRS) is one of the most widely used examiner rating instruments for screening, diagnosis and measuring the severity of depression. However, the long-term predictive value of the MADRS in patients admitted to the breast cancer diagnosis unit (BCDU) is unknown. Patients and Methods: In the Kuopio Breast Cancer Study women with breast symptoms were evaluated for the total MADRS score before any diagnostic procedures. The relapse-free survival (RFS) was calculated from the time of diagnosis to the time of first relapse including local relapse, contralateral breast cancer $(B C)$ or metastatic disease. The overall survival (OS) was assessed as the time from the date of diagnosis to the date of last follow-up or death of the patient. The effect of the MADRS on the RFS and on the OS were calculated by Kaplan-Meier survival analysis and the difference between groups was assessed by the log-rank test. The RFS and OS were estimated for the study groups with the low MADRS score $(<25)$ versus the high MADRS score $(\geq 25)$. The endpoint of our study was to determine differences in long-term outcome and in MADRS score in breast cancer $(B C)$, benign breast disease (BBD) and healthy study subjects (HSS). Results: In the Cox proportional hazard model the total MADRS score significantly predicted the 25 year RFS and $O S$ in the HSS, BBD and BC groups combined (HR=2.26, $p=0.006 ; H R=2.46, p=0.008$, respectively), and in the Kaplan-Meier survival analysis with the log-rank test the total MADRS score predicted the 25 year RFS and OS in the $H S S, B B D$ and $B C$ groups combined ( $p=0.005 ; p=0.006$,
\end{abstract}

Correspondence to: Matti Eskelinen, MD, Ph.D., School of Medicine, University of Eastern Finland, P.O. Box 100, FI-70029 KYS, Kuopio, Finland. Tel: +358 17173311, Fax: +358 17172611, GSM: +358 400969444, e-mail: matti.eskelinen@kuh.fi

Key Words: MADRS, breast disease, Breast cancer, 25-year survival. respectively). Conclusion: The moderate/severe depression (MADRS) can affect the quality of life and outcome among patients admitted to the BCDU.

Breast cancer (BC) is a common malignancy in females accounting for $15-20 \%$ of all cancer cases in women and over a half a million women die every year from BC. The worldwide differences in $\mathrm{BC}$ incidence are almost four-fold, the highest rate is in Western Europe, 96/100,000 of population, whereas in Africa the rate is 27 cases per 100,000 population population (1). In 2014, 5008 Finnish women were diagnosed with BC (2). A substantial number of well-established or suggested prognostic factors have been elaborated in the literature (3), but only very few are used in clinical decision-making in the treatment of $\mathrm{BC}$. The therapy of $\mathrm{BC}$ is based on tumor diameter, nodal status and sex steroid receptor content (3-5). Although, the prognosis of BC has improved gradually due to adjuvant hormonal therapy and chemotherapy, still too many $\mathrm{BC}$ patients experience a recurrence and therefore it would be of great importance to identify predictive factors to find the most effective treatment for each patient. In addition, subjects diagnosed with BC are confronted with a stressful situation that can result in anxiety, depression and other forms of psychological morbidity. The three recent papers indicated that $20-40 \%$ of patients with BC reported significant distress during the first year after diagnosis (6-8), although psychological distress is often unrecognized and untreated in cancer patients (9).

The Hamilton Depression Rating Scale (HAMD) and the Montgomery-Asberg Depression Rating Scale (MADRS) are the most widely used examiner-report psychometric questionnaires, which health care professionals and researchers use to measure the severity of depression in a variety of settings (10-11). Earlier, we assessed the depression and hopelessness in among patients admitted to the Breast Cancer Diagnosis Unit (BCDU) and the results indicated a highly significant agreement between different 
Table I. Characteristics of the study participants. Results are shown for the patients with breast cancer (BC), for those with benign breast disease $(B B D)$ and for the healthy study participants (HSS).

\begin{tabular}{|c|c|c|c|c|}
\hline Variable & HSS $(n=28)$ & $\mathrm{BBD}(\mathrm{n}=53)$ & $\mathrm{BC}(\mathrm{n}=34)$ & $p$-Value \\
\hline Age (mean, years) & 45.7 & 47.6 & 51.6 & 0.12 \\
\hline Height (mean, $\mathrm{cm}$ ) & 160.8 & 162.3 & 164.4 & 0.75 \\
\hline Body weight (mean, $\mathrm{kg}$ ) & 68.3 & 67.8 & 72.5 & 0.25 \\
\hline Age at menarche (mean, years) & 13.4 & 13.4 & 13.4 & 0.99 \\
\hline Age at birth of I child (mean, years) & 25.0 & 25.0 & 25.2 & 0.92 \\
\hline Age at menopause (mean, years) & 50.0 & 48.9 & 47.9 & 0.53 \\
\hline No. of children (mean) & 2.5 & 2.4 & 2.6 & 0.27 \\
\hline Parous & $23(82 \%)$ & $44(83 \%)$ & $31(91 \%)$ & 0.50 \\
\hline Breast feeding (mean, months) & 3.9 & 3.4 & 3.6 & 0.77 \\
\hline Use of oral contraceptives & $18(64 \%)$ & $25(47 \%)$ & $13(38 \%)$ & 0.12 \\
\hline HRT & $14(50 \%)$ & $36(68 \%)$ & $27(79 \%)$ & 0.44 \\
\hline Premenopausal & $18(64 \%)$ & $28(53 \%)$ & $13(38 \%)$ & 0.10 \\
\hline Postmenopausal & $10(36 \%)$ & $25(47 \%)$ & $21(62 \%)$ & 0.12 \\
\hline History of previous BBD & $10(36 \%)$ & $22(42 \%)$ & $18(53 \%)$ & 0.37 \\
\hline Family history of BC & $5(18 \%)$ & $5(9 \%)$ & $1(3 \%)$ & 0.21 \\
\hline Use of alcohol & $13(46 \%)$ & $31(58 \%)$ & $21(62 \%)$ & 0.44 \\
\hline Smoking & $10(36 \%)$ & $21(40 \%)$ & $15(44 \%)$ & 0.80 \\
\hline MADRS (mean score) & 22.2 & 21.8 & 21.2 & 0.78 \\
\hline
\end{tabular}

HRT, Use of hormonal replacement therapy.

psychometric inventories and depression scores and hopelessness measures (12-16). In addition, many studies have investigated the relationship between psychological factors, stress, adverse life events, social support, emotional control and risk of $\mathrm{BC}(17-36)$. However, there is no prospective study on whether the moderate/severe depression (MADRS) can affect the quality of life and outcome among patients admitted to the BCDU. Therefore, we carried-out a prospective study to examine the association between the MADRS, and relapse-free survival (RFS) and overall survival (OS) in 25-year cohort in Finland.

\section{Patients and Methods}

The Kuopio BC Study (KBCS) was a multidisciplinary cooperative project conducted by different departments of the University of Kuopio and Kuopio University Hospital, and included all women who were referred to the hospital for breast examination between April 1990 and December 1995. The KBCS followed the protocol of the International Collaborative Study of Breast and Colorectal Cancer coordinated by the European Institute of Oncology in Milan, and was initiated as a SEARCH program of the International Agency for Research on Cancer. The collaborative study is based on the assumption that $\mathrm{BC}$ and colorectal cancer may have common risk factors. Study centres for the BC study are situated in Canada, Finland, Greece, Ireland, Italy, Russia, Slovakia, Spain and Switzerland (38). The study participants showed BC symptoms (a lump in the breast or in the axilla, pain in the breast, bleeding from the nipple, nipple discharge or skin dimpling), or an abnormality of the breast and the indications for referral in this study were in line with our previous investigations in a BC Diagnostic Unit (BCDU) in Finland (39, 40).
This prospective case-control study was approved by the Kuopio University Hospital Board on Research Ethics (approval number 14/12/1989) and was conducted in accordance with the Declaration of Helsinki. All study subjects gave written informed consent to participate in this study. One hundred and fifteen women participated and were interviewed (to determine the level of emotional depression) by a psychiatrist (P.O.) before any diagnostic procedures, so neither the interviewer nor the patient knew the diagnosis at the time of the interview. The interviews were recorded and the ratings were completed before the final diagnosis. The clinical examination, mammography and biopsy showed BC in 34 $(29.6 \%)$ patients, BBD in $53(46.1 \%)$ patients and $28(23.4 \%)$ HSS (Table I).

Montgomery-Asberg Depression Rating Scale (MADRS). Montgomery and Asberg introduced in 1979 an inventory (MADRS) for rapid screening of depression (11). The examiner (P.O.) completed the MADRS with 10 variables and the MADRS was used as a continuos variable in this study. The questionnaire items measuring depression in the MADRS test are following; 1. apparent sadness, 2. reported sadness, 3. inner tension, 4. reduced sleep, 5. reduced appetite, 6 . concentration difficulties, 7. lassitude, 8 . inability to feel, 9. pessimistic thoughts, 10. suicidal thoughts. Each MADRS item yields a score of 0 to 6 and the MADRS test with ten variables ranges from 0 to 60 and was rated as follows: grade I, score $0(\mathrm{n}=0)$, no depression; grade II, score 1-6 $(n=25)$, few depression; grade III, score 7-19 $(n=30)$, mild depression; grade IV, score 20-34 ( $\mathrm{n}=38)$, moderate depression; and grade V, score 35-60 $(n=22)$, severe depression. In the present study the total MADRS score was used as a continuous variable with the cutoff point 25 for the total MADRS score.

Statistical analysis. Significance of the results was calculated with the SPSS/PC statistical package (SPSS Inc., Chicago, IL, USA). 


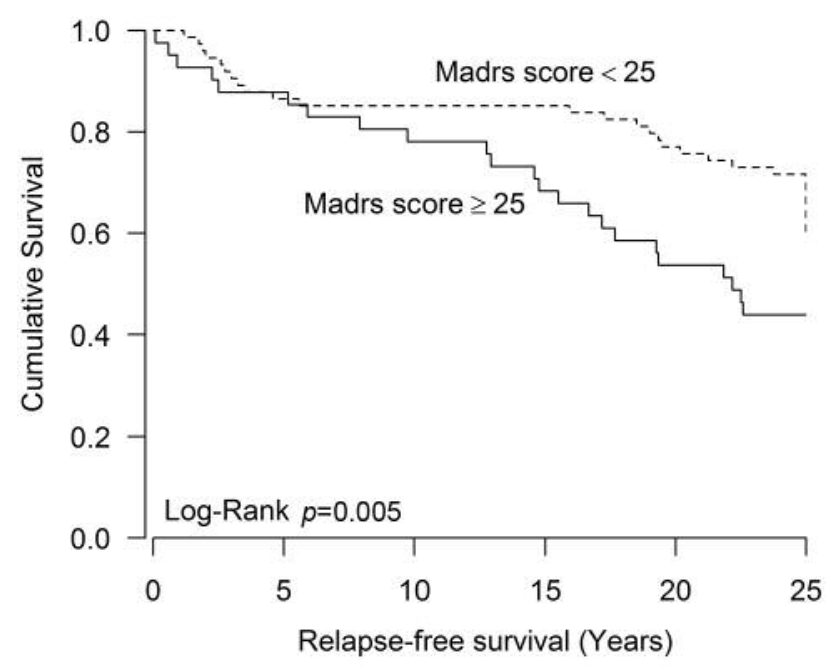

Figure 1. Kaplan-Meier survival curves for relapse-free survival (RFS) for patients with breast cancer $(B C)$, those with benign breast disease $(B B D)$ and for the healthy study participants (HSS) groups combined $(n=115)$ according to total MADRS score. The total MADRS score was a continuous variable for the study patients. The MADRS score had a statistically significant effect on RFS $(p=0.005)$ by the log-rank test.

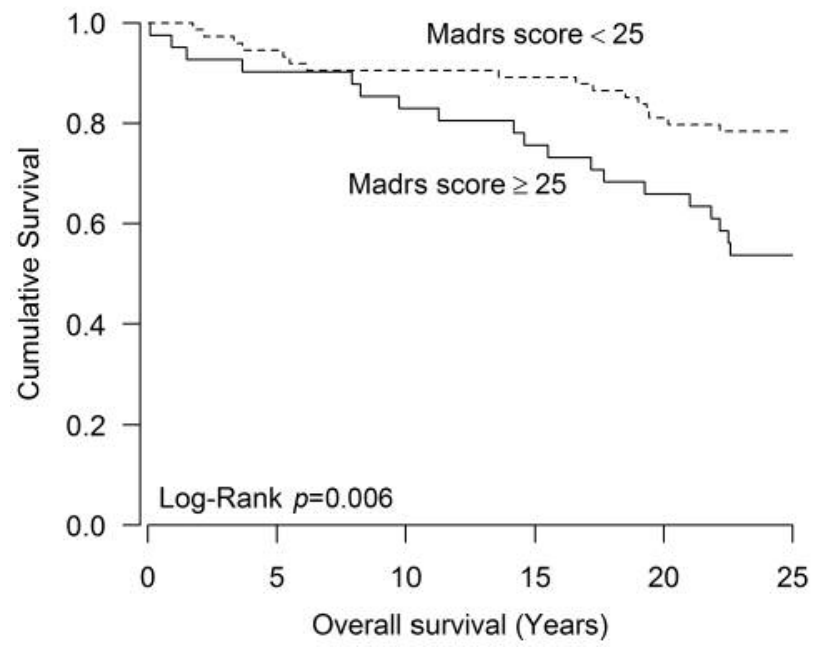

Figure 2. Kaplan-Meier survival curves for overall survival (OS) for patients with breast cancer $(B C)$, those with benign breast disease $(B B D)$ and for the healthy study participants (HSS) groups combined $(n=115)$ according to total MADRS score. The total MADRS score was a continuous variable for the study patients. The MADRS score had a statistically significant effect on OS $(p=0.006)$ by the log-rank test.
Correlations and differences between the study groups (BC, BBD and HSS groups) were measured with the two-sided chi-square test and non-parametric Kruskal-Wallis variance analyses. The data on relapse-free survival (RFS) and overall survival (OS) were collected and inspected from Kuopio University Hospital registry. The RFS was calculated from the time of diagnosis to the time of first relapse including the local relapse, the contralateral $\mathrm{BC}$ or the metastatic disease. The OS was assessed as the time from the date of diagnosis to the date of last follow-up or death of the patient. The effect of the MADRS on the RFS and on the OS were calculated by the Kaplan-Meier survival analysis and the difference between the groups was assessed by the log-rank test. The RFS and OS was estimated for the HSS, BBD and BC groups with the low MADRS score $(<25)$ versus the HSS, BBD and BC groups with the high MADRS score $(\geq 25)$. The $p$-values and the hazard ratios (HRs) and their $95 \%$ confidence intervals (CI) were calculated from the Cox proportional hazard models. The $p$-value 0.05 was considered to be statistically significant.

\section{Results}

The study groups did not differ in terms of demographic variables or surgical data. The demographic characteristics and surgical data are presented in Table I with $p$-values. Although the patients in the BC group were slightly older than those in the BBD and HSS groups (51.5 versus 47.5 and 45.7 years, respectively), the age difference was not statistically significant $(p=0.12)$. The majority of the patients $(85 / 115,74 \%)$ were married or living in a steady relationship. The mean MADRS score values were quite similar in the HSS, BBD and BC groups ( $p=0.780$, Table I) and the groups differed only slightly from each other as to the factors of the reproductive life of the women (Table I).

In Kaplan-Meier survival analysis with the difference between the groups assessed by the log-rank test, the low total MADRS score $(<25)$ showed as a statistically significant favourable predictor of the RFS (the log-rank $p=0.005$, Figure 1) and the OS (the log-rank $p=0.006$, Figure $2)$. In the Cox regression analysis the low total MADRS score $(<25)$ showed as a statistically significant favourable predictor of the RFS $(\mathrm{HR}=2.26,95 \% \mathrm{CI}=1.26-4.06, p=0.006$, Table II) and the OS (HR=2.46, 95\% CI=1.27-4.79, $p=0.008$, Table III) in the HSS, BBD and BC groups combined. A similar although statistically insignificant pattern was seen in the HSS and the BBD groups (Table II). The low total MADRS score $(<25)$ versus the high total MADRS score $(\geq 25)$ differed significantly in the 25 -year relapse rate (29.7\% vs. 56.1\%, Table II). The low total MADRS score $(<25)$ versus the high total MADRS score $(\geq 25)$ differed significantly in the 25 -year survival rate $(21.6 \%$ versus $46.1 \%$, Table III).

\section{Discussion}

$\mathrm{BC}$ is considered a hormone-dependent disease $(3,41,42)$, because a number of $\mathrm{BC}$ risk factors are related to female hormones and to a women's reproductive life such as, low parity or nulliparity, no or little breastfeeding, early age at 
Table II. Total MADRS score versus the relapse-free survival (RFS) for the HSS $(n=28), B B D(n=53)$ and $B C(n=34)$ groups and for the study groups combined. The p-values and hazard ratios (HRs) and their $95 \%$ confidence intervals (CI) were calculated from the Cox proportional hazard models.

\begin{tabular}{lccccc}
\hline Group & \multicolumn{2}{c}{ RFS $(\%)$} & HR & $95 \%$ CI & $p$-Value \\
\cline { 2 - 3 } & MADRS <25 & MADRS $\geq 25$ & & & \\
\hline All & 29.7 & 56.1 & 2.26 & $1.26-4.06$ & 0.006 \\
HSS & 31.6 & 30.0 & 0.93 & $0.23-3.72$ & 0.919 \\
BBD & 17.6 & 38.9 & 2.46 & $0.83-7.34$ & 0.106 \\
BC & 47.6 & 100.0 & 4.04 & $1.63-9.99$ & 0.003 \\
\hline
\end{tabular}

Table III. Total MADRS score versus the overall survival (OS) for the HSS $(n=28), B B D(n=53)$ and BC $(n=34)$ groups and for the study groups combined. The p-values and hazard ratios (HRs) and their $95 \%$ confidence intervals (CI) were calculated from the Cox proportional hazard models.

\begin{tabular}{lccccc}
\hline \multirow{2}{*}{ Group } & \multicolumn{2}{c}{ OS (\%) } & HR & $95 \%$ CI & $p$-Value \\
\cline { 2 - 3 } & MADRS <25 & & & \\
\hline All & 78.4 & 53.9 & 2.46 & $1.27-4.79$ & 0.008 \\
HSS & 89.5 & 70.0 & 3.00 & $0.50-17.99$ & 0.228 \\
BBD & 88.2 & 72.2 & 2.49 & $0.67-9.27$ & 0.174 \\
BC & 52.4 & 15.4 & 2.66 & $1.11-6.35$ & 0.028 \\
\hline
\end{tabular}

menarche, late age at menopause, late age at birth of first child, use of oral contraceptives and hormone replacement therapy, are known to be the main risk factors for sporadic BC (3, 41-47). Other life-style factors, such as obesity, smoking, alcohol consumption and lack of physical activity, appear to contribute to an increased risk for this malignancy, although the results concerning such factors are inconsistent (41-47). Although, there is no firm evidence for any mechanism through which psychological distress could affect the neoplasia, $\mathrm{BC}$ is a disease with great psychological impact and several mechanism have been suggested for possible psychological variables associated with BC risk (49).

Althought the MADRS test is a widely used examinerrating instrument in depression, the long-term predictive value of MADRS in patients admitted to the BCDU is unknown. The patients who have their diagnosis visit to a BCDU seem to be anxious and psychological distress can arise in the time between lump discovery, follow-up appointments due to abnormal findings, and diagnosis, regardless of whether the diagnosis is a $\operatorname{BBD}$ or $\operatorname{BC}(50,51)$. Various factors like the status of social support, marital status, educational level, and the personal psychiatric history may affect on psychological distress in BCDU patients (5153). Some studies report an association between psychological distress and clinical variables, such as type of treatment and cancer stage. In particular, studies suggest that younger cancer patients have an increased risk for psychological distress (54).

Beck depression inventory (BDI) and MADRS are the most widely used rating instruments for screening, diagnosis and measuring the status of depression. BDI is a patientrated scale and we have earlier shown that the patient-rated depression in BDI could significantly effect the long-term outcome of women in BCDU (37). The MADRS is a tenitem examiner-rated test, which health care professionals and researchers use to detect depression in normal populations and in different psychiatric patient cohorts. The MADRS was designed in 1979 by British and Swedish researchers as an adjunct to the Hamilton Rating Scale for Depression (HAMD). The MADRS gives more weight to cognitive features of depression, while the HAMD focuses more on the somatic symptoms. There is, however, a high degree of statistical correlation between scores on the two measures $(55,56)$. Muller et al. (57) estimated the MADRS cut-off scores for moderate and severe depression in relation to the Hamilton Depression Rating Scale (HAMD) and best separation between moderate and severe depression was achieved with a MADRS score of 31 (sensitivity 93.5\%, specificity $83.3 \%$ ). Higher MADRS score indicates more severe depression, and each item yields a score of 0 to 6 . The overall score ranges from 0 to 60 . However, the definition of moderate and severe depression is still a matter of debate $(58,59)$, and reliable and clinically useful gradations based on empirical data are required, since there is evidence that different new antidepressants show distinct efficacy depending on the severity of illness (58-60).

From the methodological point of view, the limitation of our study is its small sample size of 115 patients. However, this research question is very specific and therefore we did not expect high number of patients. This could be taken in account when planning new scientific studies of the depression scoring in long-term outcome in the $\mathrm{BC}$ patients in the future. In all the study groups, the study subjects were assessed by a psychiatrist; i.e. all ratings of an individual patient were carried out by the same rater and the MADRS score was used as a continuous variable with the cut-off point 25 for the total MADRS score. Therefore, it is unlikely that there is a study bias from the MADRS scoring between the three study groups. Other methodological point, which needs to be further discussed is the possible downsides of MADRS test when used in patients regarding its longer-term predictive capability. HAMD and MADRS differ in their coverage/number of depressive items and the symptoms of atypical depression like hypersomnia or appetite/weight increase are not included. Symptoms of motor retardation are 
not covered by the MADRS (61).The most frequently used depression rating scales (HAMD, BDI and MADRS) are still considered as 'gold standards'. However, the ability of each scale to differentiate between healthy (HSS), benign (BBD) and breast cancer patients $(\mathrm{BC})$ and to detect changes during treatment or follow-up may differ in various stages of $\mathrm{BC}$. Therefore, BC patients should have regular follow-up visits in order to identify mental health problems well in time (62).

In conclusion, the results suggest that the MADRS score used as a continuous variable with the cut-off point 25 for the total MADRS score significantly correlates to the 25-year RFS and OS in the HSS, BBD and BC groups.

\section{Conflicts of Interest}

None.

\section{Acknowledgements}

This study was funded by the Heikki, Aino and Aarne Korhonen foundation, Paavo Koistinen Foundation, and EVO funds from Kuopio University Hospital. Our special thanks are due to Ms AnnaKaisa Lyytinen, R.N. for help in data collection.

\section{References}

1 Bray F, Ren JS, Masuyer E and Ferlay J: Global estimates of cancer prevalence for 27 sites in the adult population in 2008 . Int J Cancer 132: 1133-1145, 2013.

2 Finnish Cancer Registry. Cancer Statistics at www.cancerregistry.fi, 2014.

3 Aaltomaa S, Lipponen P, Eskelinen M, Kosma VM, Marin S, Alhava E and Syrjänen K: Hormone receptors as prognostic factors in female breast-cancer. Ann Med 23: 643-648, 1991.

4 Jatoi I, Hilsenbeck SG, Clark GM and Osborne CK: Significance of axillary lymph node metastasis in primary breast cancer. $\mathrm{J}$ Clin Oncol 17: 2334-2340, 1999.

5 Elkin EB, Hudis C, Begg CB and Schrag D: The effect of changes in tumor size on breast carcinoma survival in the U.S.:1975-1999. Cancer 104: 1149-1157, 2005.

6 Golden-Kreutz DM and Andersen BL: Depressive symptoms after breast cancer surgery: Relationships with global, cancerrelated, and life event stress. Psychooncology 13: 211-220, 2004.

7 Bardwell WA, Natarajan L, Dimsdale JE, Rock CL, Mortimer JE, Hollenbach K and Pierce JP: Objective cancer-related variables are not associated with depressive symptoms in women treated for early-stage breast cancer. J Clin Oncol 24: 24202427, 2006.

8 Hegel MT, Moore CP, Collins ED, Kearing S, Gillock KL, Riggs RL, Clay KF and Ahles TA: Distress, psychiatric syndromes, and impairment of function in women with newly diagnosed breast cancer. Cancer 107: 2924-2931, 2006.

9 Fallowfield L, Ratcliffe D, Jenkins V and Saul J: Psychiatric morbidity and its recognition by doctors in patients with cancer. Br J Cancer 84: 1011-1105, 2001.

10 Hamilton M: A rating scale for depression. J Neurol Neurosurg Psychiatry 23: 56-62, 1960.
11 Montgomery SA and Asberg M: A new depression scale to be sensitive to change. Br J Psychiatry 134: 382-389, 1979.

12 Eskelinen $\mathrm{M}$ and Ollonen P. Measurement of pessimism: hopelessness scale in healthy study subjects, and in patients with benign breast disease and breast cancer: A prospective casecontrol study in Finland. Anticancer Res 31: 4019-4023, 2011.

13 Eskelinen M, Korhonen R, Selander T and Ollonen P: The SelfRating Score (SRS) versus the Examiner Rating Score (ERS) in measuring helplessness in healthy individuals and in patients with benign breast disease and breast cancer: a prospective casecontrol study in Finland. Anticancer Res 34: 5677-5682, 2014.

14 Eskelinen M, Korhonen R, Selander T and Ollonen P: The relation of hopelessness/helplessness versus Beck Depression Inventory (BDI) in healthy individuals and in patients with benign breast disease and breast cancer: a prospective casecontrol study in Finland. Anticancer Res 35: 941-948, 2015.

15 Eskelinen $\mathrm{M}$, Korhonen $\mathrm{R}$, Selander $\mathrm{T}$ and Ollonen $\mathrm{P}$ : Agreement between Hopelessness/Helplessness versus Montgomery-Asberg depression rating scale (MADRS) in healthy individuals and in patients with benign breast disease and breast cancer: a prospective case-control study in Finland. Anticancer Res 35: 2215-2222, 2015.

16 Eskelinen M, Korhonen R, Selander T and Ollonen P: Suicidal and pessimistic thoughts versus hopelessness/helplessness in healthy individuals and in patients with benign breast disease and breast cancer: a prospective case-control study in Finland. Anticancer Res 35: 3543-3545, 2015

17 Chen CC, David AS, Nunnerley H, Michell M, Dawson JL, Berry H, Dobbs J and Fahy T: Adverse life events and breast cancer: case-control study. BMJ 311: 1527-1530, 1995.

18 Roberts FD, Newcomb PA, Trentham-Dietz A and Storer BE: Self-reported stress and risk of breast cancer. Cancer 77: 10891093, 1996.

19 Protheroe D, Turvey K, Horgan K, Benson E, Bowers D and House A: Stressful life events and difficulties and onset of breast cancer: case-control study. BMJ 319: 1027-1030, 1999.

20 Price MA, Tennant CC, Butow PN, Smith RC, Kennedy SJ, Kossoff MB and Dunn SM: The role of psychosocial factors in the development of breast carcinoma: Part II. Life event stressors, social support, defense style, and emotional control and their interactions. Cancer 91: 686-697, 2001.

21 Duijts SFA, Zeegers MPA and VD Borne B: The association between stressful life events and breast cancer risk: a metaanalysis. Int J Cancer 107: 1023-1029, 2003.

22 Ollonen P, Lehtonen J and Eskelinen M: Stressful and adverse life experiences in patients with breast symptoms; a prospective case-control study in Kuopio, Finland. Anticancer Res 25: 531$536,2005$.

23 Ollonen P, Lehtonen J and Eskelinen M: Anxiety, depression and the history of psychiatric symptoms in patients with breast disease: a prospective case-control study in Kuopio, Finland. Anticancer Res 25: 2527-2534, 2005.

24 Ollonen P, Lehtonen J and Eskelinen M: Coping and defending as risk factors for breast cancer in patients with breast disease: a prospective case-control study in Kuopio, Finland. Anticancer Res 25: 4623-4630, 2005.

25 Ollonen $\mathrm{P}$ and Eskelinen M: Idealization as risk factor for breast cancer in patients with breast disease: a prospective casecontrol study in Kuopio, Finland. Anticancer Res 27: 16251630, 2007. 
26 Eskelinen M and Ollonen P: Psychosocial risk scale (PRS) for breast cancer in patients with breast disease: a prospective casecontrol study in Kuopio, Finland. Anticancer Res 29: 4765-4770, 2009.

27 Eskelinen $\mathrm{M}$ and Ollonen P: The body image drawing analysis in women with breast disease and breast cancer: anxiety, colour and depression categories. Anticancer Res 30: 683-691, 2010.

28 Eskelinen $\mathrm{M}$ and Ollonen P:Evaluation of women with breast disease using body image drawing analysis. Anticancer Res 30: 2399-2406, 2010.

29 Eskelinen M and Ollonen P: Life stress due to losses and deficit in childhood and adolescence as breast cancer risk factor: a prospective case-control study in Kuopio, Finland. Anticancer Res 30: 4303-4308, 2010.

30 Eskelinen M, and Ollonen P: Life stress and losses and deficit in adulthood as breast cancer risk factor: a prospective casecontrol study in Kuopio, Finland. In Vivo 24: 899-904, 2010.

31 Eskelinen M and Ollonen P: Beck Depression Inventory (BDI) in patients with breast disease and breast cancer: a prospective case-control study. In Vivo 25: 111-116, 2011.

32 Eskelinen $\mathrm{M}$ and Ollonen P: Forsen Psychological Risk Inventory for breast cancer patients: a prospective case-control study with special reference to the use of psychiatric medications. Anticancer Res 31: 739-744, 2011.

33 Eskelinen $\mathrm{M}$ and Ollonen P: Montgomery-Asberg depression rating scale (MADRS) in healthy study subjects, in patients with breast disease and breast cancer: a prospective case-control study. Anticancer Res 31: 1065-1069, 2011.

34 Eskelinen $\mathrm{M}$ and Ollonen P: Assessment of general anxiety in patients with breast disease and breast cancer using the Spielberger STAI self evaluation test: a prospective case-control study in Finland. Anticancer Res 31: 1801-1806, 2011.

35 Eskelinen M, and Ollonen P: Sifneos Alexithymia Questionnaire in assessment of general alexithymia in patients with breast disease and breast cancer: a prospective case-control study in Finland. Anticancer Res 31: 3101-3106, 2011.

36 Eskelinen $\mathrm{M}$ and Ollonen P: Assessment of 'cancer-prone personality' characteristics in healthy study subjects and, in patients with breast disease and breast cancer using the Commitment Questionnaire: a prospective case-control study in Finland. Anticancer Res 31: 4013-4017, 2011.

37 Eskelinen M, Korhonen R, Selander T and Ollonen P: Beck Depression Inventory as a predictor of long-term outcome among patients admitted to breast cancer diagnosis unit: a 25year cohort study in Finland. Anticancer Res 37: 819-824, 2017.

38 Boyle P: SEARCH programme of the International Agency for Research on Cancer. Eur J Cancer 26: 547-549, 1990.

39 Eskelinen MJ, Pajarinen P, Collan Y, Pesonen E, Alhava E, Kettunen K and Nordling S: Relationship between DNA ploidy and survival in patients with primary breast cancer. Br J Surg 76: 830-834, 1989.

40 Eskelinen MJ, Lipponen PK, Collan Y and Syrjänen KJ: The role of nucleolar organizer regions as prognostic factors in breast cancer. Eur J Cancer 27: 989-992, 1991.

41 Aaltomaa S, Lipponen P, Eskelinen M, Kosma VM, Marin S, Alhava E and Syrjänen K: Prognostic scores combining clinical, histological and morphometric variables in assessment of the disease outcome in female breast cancer. Int J Cancer 49: 886$892,1991$.
42 Key JA, Verkasalo PK and Banks E: Epidemiology of breast cancer. Lancet Oncol 2: 133-140, 2001.

43 Zhu ZR, Agren J, Männistö S, Pietinen P, Eskelinen M, Syrjanen $\mathrm{K}$ and Uusitupa M: Fatty-acid composition of breast adiposetissue in breast cancer patients and in patients with benign breast disease. Nutr Cancer 24: 151-160,1995.

44 Pietiläinen T, Lipponen P, Aaltomaa S, Eskelinen M, Kosma V$\mathrm{M}$ and Syrjänen $\mathrm{K}$ : Expression of retinoblastoma gene protein (RB) in breast cancer as related to established prognostic factors and survival. Eur J Cancer 31: 329-333, 1995.

45 Pellikainen J, Kataja V, Ropponen K, Kellokoski J, Pietiläinen T, Böhm J, Eskelinen M and Kosma VM: Reduced nuclear expression of transcription factor AP-2 associates with aggressive breast cancer. Clin Cancer Res 8: 3487-3495, 2002.

46 Mitrunen K, Kataja V, Eskelinen M, Kosma VM, Kang D, Benhamou S, Vainio H, Uusitupa M and Hirvonen A: Combined COMT and GST genotypes and hormone replacement therapy associated breast cancer risk. Pharmacogenetics 12: 67-72, 2002.

47 Metsola K, Kataja V, Sillanpää P, Siivola P, Heikinheimo L, Eskelinen M, Kosma VM, Uusitupa M and Hirvonen A: XRCC1 and XPD genetic polymorphisms, smoking and breast cancer risk in a Finnish case-control study. Breast Cancer Res 7: R987997, 2005.

48 Sillanpää P, Hirvonen A, Kataja V, Eskelinen M, Kosma V-M, Uusitupa M, Vainio H and Mitrunen K: NAT2 slow acetylator genotype as an important modifier of breast cancer risk. Int $\mathrm{J}$ Cancer 114: 579-584, 2005.

49 Hilakivi-Clarke L: Estrogen-regulated non-reproductive behaviors and breast cancer risk: animal models and human studies. Breast Cancer Res Treat 46: 143-159, 1997.

50 Drageset S and Lindstrom TC: The mental health of women with suspected breast cancer: the relationship between social support, anxiety, coping, and defense in maintaining mental health. J Psychiatr Ment Health Nurs 10: 401-409, 2003.

51 Ando N, Iwamitsu Y, Kuranami M, Okazaki S, Nakatani Y, Yamamoto $\mathrm{K}$, Watanabe $\mathrm{M}$ and Miyaoka $\mathrm{H}$ : Predictors of psychological distress after diagnosis in breast cancer patients and patients with benign breast problems. Psychosomatics 52: 56-64, 2011.

52 Eskelinen $\mathrm{M}$ and Ollonen P: Contribution of emotional distance and reserve in patient-physician communication in healthy study patients, and in patients with benign breast disease and breast cancer: a prospective case-control study in Finland. Anticancer Res 34: 1269-1274, 2014.

53 Eskelinen M, Korhonen R, Selander T and Ollonen P: Emotional personality/proximity versus emotional authenticity in patientphysician communication in healthy study participants, and in patients with benign breast disease and breast cancer: a prospective case-control study in Finland. Anticancer Res 35: 1627-1634, 2015.

54 Cohee AA, Stump T, Adams RN, Johns SA, Von Ah D, Zoppi K, Fife B, Monahan PO, Cella D and Champion VL: Factors associated with depressive symptoms in young long-term breast cancer survivors. Qual Life Res 25: 1991-1997, 2016.

55 Maier W, Philipp M, Heuser I, Schlegel S, Buller R and Wetzel $\mathrm{H}$ : Improving depression severity assessment-I. Reliability, internal validity and sensitivity to change of three observer depression scales. J Psychiatr Res 22: 3-12, 1988.

56 Ahmadpanah M, Sheikhbabaei M, Haghighi M, Roham F, Jahangard L, Akhondi A, Sadeghi Bahmani D, Bajoghli H, 
Holsboer-Trachsler E and Brand S: Validity and test-retest reliability of the Persian version of the Montgomery-Asberg Depression Rating Scale. Neuropsychiatr Dis Treat 12: 603-607, 2016.

57 Muller MJ, Himmerich H, Kienzle B and Szegedi A: Differentiating moderate and severe depression using the Montgomery-Åsberg depression rating scale (MADRS). J Affect Dis 77: 255-260, 2003.

58 Hirschfeld RM:American health care systems and depression: the past, present, and the future. J Clin Psychiatry 59(Suppl 20): 5-10, 1998.

59 Schatzberg AF: Antidepressant effectiveness in severe depression and melancholia. J Clin Psychiatry 60(Suppl 4): 14$21,1999$.
60 Kienke AS and Rosenbaum JF: Efficiency of venlaflaxine in the treatment of severe depression. Depression Anxiety 12: 50-54, 2000.

61 Demyttenaere K and De Fruyt J: Getting what you ask for: on the selectivity of depression rating scales. Psychother Psychosom 72: 61-70, 2003.

62 Fafouti M, Paparrigopoulos T, Zervas Y, Rabavilas A, Malamos N, Liappas I and Tzavara C: Depression, anxiety and general psychopathology in breast cancer patients: a cross-sectional control study. In Vivo 24: 803-810, 2010.

Received March 2, 2017

Revised March 15, 2017

Accepted March 20, 2017 